\title{
Fast Parallel Network Packet Filter System based on CUDA
}

\author{
Che-Lun Hung* \\ Department of Computer Science and Communication Engineering, Providence University, 200, sec. 7, Taiwan \\ Boulevard, Shalu Dist., \\ Taichung, 43301, Taiwan \\ E-mail: clhung@pu.edu.tw \\ Shih-Wei Guo \\ Department of Computer Science and Information Managemen, Providence University, 200, sec. 7, Taiwan Boulevard, \\ Shalu Dist., \\ Taichung, 43301, Taiwan \\ E-mail:cometlcc@gmail.com
}

\begin{abstract}
In recent years, with the rapid development of the network hardware and software, the network speed is enhanced to multi-gigabit. Network packet filtering is an important strategy of network security to avoid malicious attacks, and it is a computation-consuming application. Therefore, we develop two efficient GPGPU-based parallel packet classification approaches to filter packets by leveraging thousands of threads. The experiment results demonstrate that the computational efficiency of filtering packet can be significantly enhanced by using GPGPU.
\end{abstract}

Keywords: Packet filtering; Packet classification; GPGPU; CUDA; Parallel Processing.

\section{Introduction}

In the past few years, the development of network bandwidth and hardware technologies have grown rapidly, a variety of Internet services have been popular, such as email system, storage system, entertainment system and others. Currently, many famous corporations, such as Google, Amazon, IBM an d Microsoft have released a variety of cloud services including hardware, software and platform. These services deeply rely on Internet. To maintain the robustness of Internet environments, the network security is one of the important parts indeed. Therefore, Internet security has become an important role to protect activities on Internet. For this purpose, packet analysis ${ }^{1}$ is useful strategy to control that packet data can flow to and from a network. The criteria t hat use when inspecting packets are based on the Layer 3 (IPv4 and IPv6) and Layer 4 (TCP, UDP, ICMP, and ICMPv6) headers. The commonly used criteria are s ource and destination address, source and destination port and protocol.

Packet analysis is at the core of timely detection and typically relies o $\mathrm{n}$ a $\mathrm{p}$ acket filtering system. P acket filtering system drops the packets if packets match to the filter rules. A packet is compared with the filter rules in sequential order from the first to the last. Until

*Corresponding author: Che-Lun Hung. E-Mail: clhung@pu.edu.tw. 
the packet matches a rule containing the keyword such as port number or IP address, the packet will be compared against all filter rules before the final action is taken. The last matched rule dictates what action to take on the packet. There is an i mplicit pass all at $t$ he beginning of a filtering rule set meaning that if a packet does not match any filter rule the resulting action will be passed. In general, each incoming packet can be considered independently of any other packet. Although for the IP fragments, the first fragment is related to other fragments apparently but arriving without order. It can be considered as independent for classification ${ }^{2}$.

Packet filter system also has been applied to many network intrusion detection systems (NIDS) as the first stage. Botnet has become one major threat to In ternet users in recent years. A botnet consists of a larg e number of bots that are $\mathrm{n}$ etworked computers compromised by malicious attackers. In general, an attacker conducts the bots to launch a variety of types of attacks such as phishing and spamming with a botnet, and then receives benefits from a variety of aspects such as economy and social security. Most of methods to detect bot's activities according to predefined patterns and signatures retrieved from well-known bots $3,4,5,6,7,8$. Although signature-based approaches are able to detect bots accurately, it is difficult to detect botnet in real time. Nowadays, detecting signature becomes more and more complex because the number of attacks is increased dramatically, and the signatures also become sophisticated. In addition, busy-hour Internet traffic will increase by a factor of 3.4 between 2013 an d 2018 . Therefore, to analyze the entire traffic on a high-speed link is computation-consuming problem. Consequently, random packet losses are likely to occur if the network traffic exceeds the capacity of packet filter of the botnet detection system. These botnet detection approaches has a very important stage, traffic reduction, to reduce the data set to the meaningful subset of flows to speed later stages in these approaches. To reduce the network traffic, packet filtering is useful strategy to control that packet data can flow to and from a network.

Sequential-process packet classifiers often take longer to clas sify a pack et set captured off a g iga-bit network interface than it took the set to arrive, making them infeasible for real-time traffic analysis ${ }^{4}$. To solve packet filter problem leaded by large amounts of traffic, one of the solutions is to in crease the processing capacity of the botnet detection system. It can be either hardware $^{9}$ or software ${ }^{10}$ solutions. However, the cost of these solutions is huge. The reasonable cost of available computing power to anal yze the network traffic is critical. Therefore, parallel process is useful solution to improve the performance of the detection system.

Recently, many literatures tried to use GeneralPurpose Graphics Processing Unit (GPU) to s olve computation intensive problem of various domains $^{11,12,13,14,15,16,17}$. GPGPU programming has been successfully utilized in the scientific computing domains which involve a high level of numeric computation. However, other applications also could be successfully parallelized by GPGPU. The greatest benefit is that the processing units grow from many (CPU, about 2-16) to massive (GPU, about over 512). In 2006, NVIDIA proposed the Compute Unified Device Architecture (CUDA). CUDA uses a n ew computing architecture named Single Instruction Multiple Threads $(\mathrm{SIMT})^{18}$. This architecture allows thread to ex ecute independent and divergent instruction streams, facilitating decision-based execution that is not provide for by the more common Single Instruction Multiple Data (SIMD).

To improve the computational performance of the Botnet detection system, we propose two efficient network traffic reduction algorithms to filter packets simultaneously by using GPGPU device. By leveraging nVidia CUDA device can achieve low cost, commodity GPU co-processors to accelerate packet-filtering throughput. The proposed algorithms could be utilized in any botnet detection method that adopts traffic reduction strategy. We al so implement the proposed packet classification algorithm on a variety of memory architectures on GPU to dis cuss the performance of proposed method. The experiment results demonstrate that the proposed method can achieve over $20 \mathrm{t}$ imes speed up over the sequential packet classification software on single CPU. It $p$ resents that GPGPU is useful for real-time traffic analysis.

\section{Related Works}

According the previous literature ${ }^{1}$, the classification can be categorized into three types: IP routing, packet demultiplexing and packet analysis. The slight differences of these types are bet ween target environments. IP routing is utilized to forward incoming packets through the correct in terface to a des tination host. Packet demultiplexing is concerned that 
forwarding the packets to the next hop router, or dropping the packets altogether. Packet analysis is similar in many respects to demultiplexing, and often depends on similar filtering algorithms, but may process a wider variety of packets, with a broader ran ge of destinations. All of these three types are depending on the packet filtering. A filter is a predicate function that operates over a collection of criteria to com pare each arriving packet ${ }^{1,19,20}$. Generally, a packet is classified by a filter which has the specific criteria. Filter criteria are Boolean valued comparisons, performed between values contained in discreet bit-ranges in the packet header and static protocol defined values.

The commonly-used and most reliable methods of classifying packet data are exhaustive search algorithms which compare packets against each and every filter in the filter set $u$ ntil a ex act match is $f$ ound ${ }^{1,21}$. These algorithms are generally slow, and thus not very useful. Other classes of algorithms include decision tree, decomposition and tuple space approaches. Decision tree algorithms are diverse in design, but all leverage a sequential tree li ke traversal of a sp ecialized data structure in order to narrow down the number of criteria against which the packet need to be compared ${ }^{1,19,22,23}$. Most of demultiplexing and analysis filters are highly sequential approaches based on decision trees ${ }^{19,20,24}$, and thus are suitable to the processing on CPUs. In contrast, decomposition algorithms can be equipped on parallel processing hardware such as FPGAs, typically splitting filter classifications into smaller sub-classifications which can be perf ormed in parallel ${ }^{1,25,26}$. Tuple space algorithms are highly specialized, and exploit a variety of filter set $\mathrm{p}$ roperties in order to reduce processing time $^{1}$.

Most of demultiplexing algorithms adopt decis ion tree approaches because of their efficiency at pru ning redundant computation on sequential processors. BPF is a well known algorithm that adopted Control Flow Graphs (CFGs) in a $\mathrm{n}$ assembler style programmable pseudo-machine to improve performance on registerbase processor ${ }^{20}$. Mach Packet Filter (MP F) and Dynamic Packet Filter (DPF) are ex tended from BPF. MPF was designed to extend and improve demultiplexing performance ${ }^{27}$, while DPF focused on exploiting dynamic code generation in order to prune redundant instructions ${ }^{24}$. These filters led to the development of $\mathrm{BPF}+{ }^{19}$, which adopted techniques such as Predicate Assertion Propagating and Partial
Redundancy Elimination, in conjunction with Just-InTime (JIT) processing and various other optimizations, to dramatically improve processing speeds. Extensible Packet Filter (xPF) ${ }^{28}$, Fairly Fast Packet Filter (FFPF) ${ }^{29}$ and $\mathrm{SWIFT}^{30}$, were developed to reduce the context switching overhead, facilitate high performance demultiplexing between multiple network monitoring application, and reduce filter update latency to support real-time filter updates, respectively.

Presently, RFC algorithm ${ }^{31}$, which is a generalization of cross-producting ${ }^{32}$, is the fastest classification algorithm in terms of the worst-case performance. Bitmap compression has been used in IPv4 forwarding ${ }^{33,34}$ and IPv6 forwarding ${ }^{35}$. It is applied to classification to compress redundant storage in data structure $^{36}$. However, the performance bottleneck of these methods are searching the compressed tables, and thus additional techniques have to be introduced to improve the inefficiency of calculating the number of bits set in a b itmap. Lulea ${ }^{33}$ algorithm utilizes a summary array to pre-process the number of bits set in the bitmap, and thus it needs an extra memory access operation per trie-node to search the compressed table. The Bitmap- $\mathrm{RFC}^{37}$ employs a built-in bit-manipulation instruction to calculate the number of bits set at runtime and apply bitmap compression to reduce its memory requirement to solve the problem of memory explosion. Thus, it is much more efficient than Lulea's in terms of time and space complexity.

However, these sequential packet classification algorithms take longer to clas sify a packet set captured off a giga-bit network interface than it to ok the set to arrive, making them infeasible for real-time traffic analysis.

Nottingham et al., ${ }^{2,11}$ proposed a clas sification algorithm, by utilizing GPU co-processors to accelerate classification throughput and maximize processing efficiency in highly parallel execution context. They provided valuable articles for introducing the concept of parallel packet classification on CUDA and OpenCL platforms. However, these literatures are lack of the performance comparisons and implementations with a variety of memory architectures of GPU. Han et al., ${ }^{38}$ proposed a GPU-based IP routing approach named PacketShader. The experiment results show that GPUbased IP routing algorithm can enhance the performance over the CPU-based IP routing approaches. Hung et al., ${ }^{39}$ presented a GP U-based network packet pattern- 
matching algorithm for network intrusion detection systems by leveraging the computational power of GPUs to accelerate pattern-m atching operations and subsequently increase the overall processing throughput. The experimental results showed that their algorithm can achieve a maximal traffic processing throughput of over $2 \mathrm{Gbit} / \mathrm{s}$. These articles present an alternative of developing the parallel $\mathrm{p}$ acket classification by leveraging GPU devices.

\section{CUDA Programming Model}

As the GPU has become increasingly more powerful and ubiquitous, researchers have begun developing various non-graphics, or general-purpose applications ${ }^{13}$. Generally, the GPUs are organized in a streaming, dataparallel model in which the co-processors execute the same instructions on multiple data streams simultaneously. Modern GPUs include several (tens to hundreds) of types of stream processors, both of graphical and general-purpose applications thus are faced with parallelization challenges in using GPUs ${ }^{41}$.

nVidia released the Compute Unified Device Architecture (CUDA) SDK to as sist developers in creating non-graphics applications that run on GPUs. A CUDA programs typically consist of a component that runs on the $\mathrm{CPU}$, or h ost, and a sm aller but computationally intensive component called the kernel that runs in parallel on the GPU. Input data for the kernel must be copied to the GPU's on-board memory from host's main memory through the PCI-E bus prior to invoking the kernel, and output data also should be written to the GPU's memory first before copying to host's main memory. All memory used by the kernel should be pre-allocated.

Kernel executes a collecti on of threads that computes a res ult for a s mall segment of data. $\mathrm{T} o$ manage multiple threads, kernel is $p$ artitioned into thread blocks, with each thread block being limited to a maximum of 512 threads. The thread blocks are usually positioned within a one or two-dimensional grid. Each thread can be positioned within a given block where it belongs, and this given block can be positioned within the grid. Therefore, each thread can calculate which elements of data to operate on, and which regions of memory to write output to by an algebraic formula. Each block is executed by a sin gle multiprocessor, which allows all $\mathrm{t}$ hreads within the block to

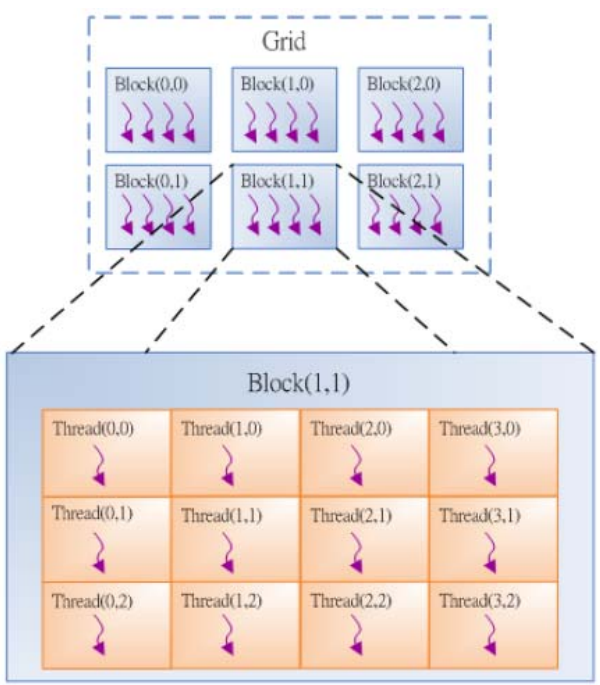

Fig. 1. The parallelism architecture of CUDA.

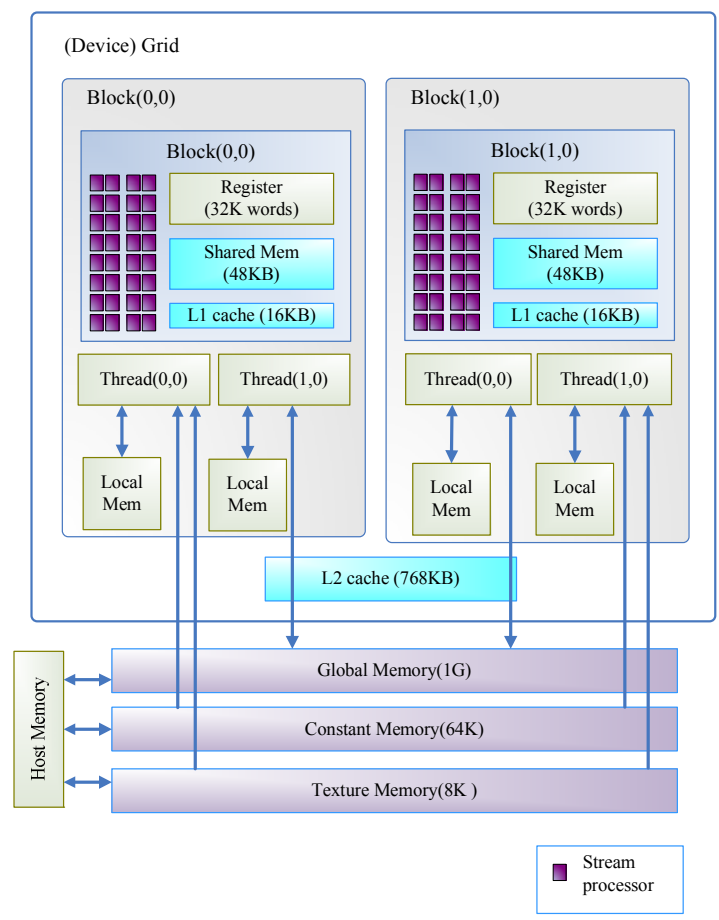

Fig. 2. Memory architecture of CUDA device, nVidia GTS450.

communicate through on-chip shared memory. The parallelism architecture of GPGPU is illustrated in Fig. 1. 
Table 1. Overview of memory architectures in CUDA device.

\begin{tabular}{l|l|l|l|l|l}
\hline Memory & Scope & Hardware & Latency & Bandwidth & Access \\
\hline Register & Thread & Chip & Immediate & & R/W \\
Shared & Block & Chip & $4-6$ clock & $200 \mathrm{~GB} / \mathrm{s}$ & $\mathrm{R} / \mathrm{W}$ \\
Constant & Grid & Cache & $4-600$ clock & $200-300 \mathrm{~GB} / \mathrm{s}$ & $\mathrm{R}$ \\
Texture & Grid & Cache & $4-600$ clock & $200-300 \mathrm{~GB} / \mathrm{s}$ & $\mathrm{R}$ \\
Global & Grid & DRAM & $400-600$ clock & $100 \mathrm{~GB} / \mathrm{s}$ & $\mathrm{R} / \mathrm{W}$ \\
Local & Thread & DRAM & $400-600$ clock & $100 \mathrm{~GB} / \mathrm{s}$ & $\mathrm{R} / \mathrm{W}$ \\
\hline
\end{tabular}

CUDA devices provide access to s everal memory architectures, such as global memory, constant memory, texture memory, share memory and registers, with their access latencies and limitations. The performance of device is relevant to the memory variants. Figure 2 illustrates the memory architectures of CUDA device. Table 1 shows the characteristics of memory architectures.

- Global Memory: Global memory is the biggest memory region available on CUDA devices and is capable of storing hundreds of megabytes of data. However, the access latency is highest than others.

- Constant Memory: Constant memory is a small read-only memory region that resides in DRAM on CUDA device. It is globally accessible memory for all threads. Since Constant memory has on-chip cache, the access latency is low.

- Texture Memory: Texture memory is a compromise between global and constant memory. Each multiprocessor on the CUDA device equips a $64 \mathrm{~K} \mathrm{~B}$ texture cache which can be bound to on e or more arbitrarily sized region of global memory. Texture memory is read only as constant memory.

- Register: Each thread block in CUDA device equips a regis ter file that contains registers. The register provides fast thread-local storage during kernel execution.

- Shared Memory: Shared memory is block-local that facilitates cooperation between multiple threads in an executing thread block. The access latency of shared memory is equivalent to that of register.

The G80 that introduced the CUDA architecture had $86.4 \mathrm{~GB} / \mathrm{s}$ of memory bandwidth, plus an $8 \mathrm{G} \mathrm{B} / \mathrm{s}$ communication bandwidth with the CPU. A CUDA application can transfer data from the system memory at $4 \mathrm{~GB} / \mathrm{s}$ and at the same time upload data back to the system memory at $4 \mathrm{~GB} / \mathrm{s}$. altogether, there is a combined total of $8 \mathrm{~GB} / \mathrm{s}$. the communication bandwidth is much lower than the memory bandwidth and may seem like a li mitation. Two memory transmission models, memory direct copy and zerocopy, in CUDA architecture is used to copy data from system memory to device memories including global, constant, texture and registers.

The CUDA runtime system provides application programming interface (API) functions to allocate an d release memory on the device and transfer pertinent data from the host memory to the allocated device memory. It could be the bottleneck of GPU computing performance. The zerocopy function is a feature that was added i $\mathrm{n}$ version $2.2 \mathrm{of}$ the CUDA Toolkit. It enables GPU threads to dire ctly access host memory. The zerocopy function can be used in place of streams because kernel-originated data transfers automatically overlap kernel execution without the overhead of setting up and determining the optimal number of streams. Different to previous two memory transfer models, streaming model is another way to improve the use of the threads and data trans fer. It is a pipelin e of asyconizing the data transmission. A stream is a sequence of operations that are performed in order on CUDA device. Figure 3 shows the streaming model of CUDA architecture. In Fig. 3, data is copied from host memory to device memory and stream 1 then lunches kernel function 1 , and when stream 1 is copying output data from device memory to host memory and stream 2 starts to lunch kernel function 2. The processes for stream 3 and stream 4 are as same as pervious processes of stream 1 and stream 2.

\section{Method}


Che-Lun Hung et al.

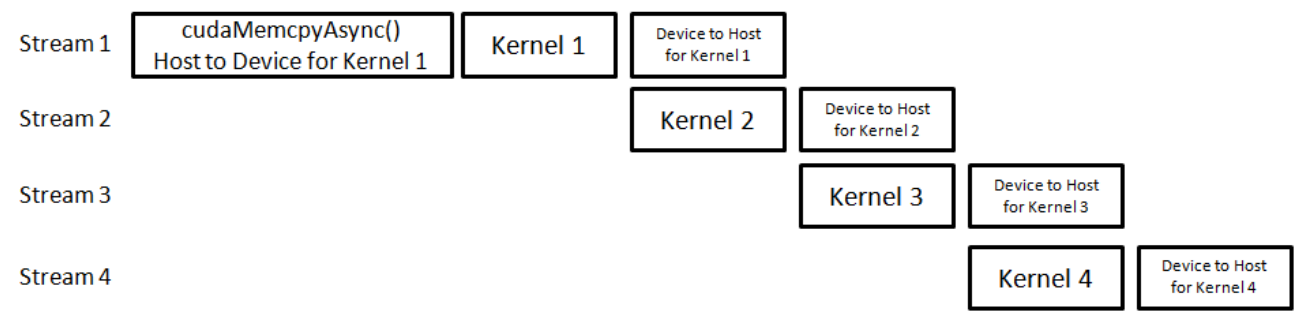

Fig. 3. Streaming approach on CUDA.

In CUDA devices, each phy sical multiprocessor contains only a single instruction register which drives eight independent processing cores simultaneously. Therefore, any divergence between thread executing on the same multiprocessor forces the instruction register to issue instruction for all th read paths sequentially whilst non-participating thread sleep ${ }^{18}$. The significant thread divergence can dramatically impair performance. To avoid thread divergence, each thread should process the similar length of data. In a filter set, the rules of each filter have a various number of fields. Due to this reason, we restrict that he number of fields of each rule should be the same at the same filter. Table 2 presents the revised filter rules. The filter sets are stored in constant memory or register files, and the packet data is stored in global memory or texture memory. Figure 4 illustrates the memory usage for filter sets sto red in constant memory and packet data stored in global memory on CUDA device. A thread process a packet data according the filter sets. Currently, we include two famous filter classification algorithms, BPF and BitMap-RFC, in the proposed method. We ch ose these two algorithms because they are totall y different data s tructures and well-known algorithm. BPF uses data structure as decision tree $\mathrm{s}$ tructure and BitMap-RFC uses data structure as hash table. $\mathrm{T}$ hrough different data structures, it can be measured that which data structure is suitable for GPU.

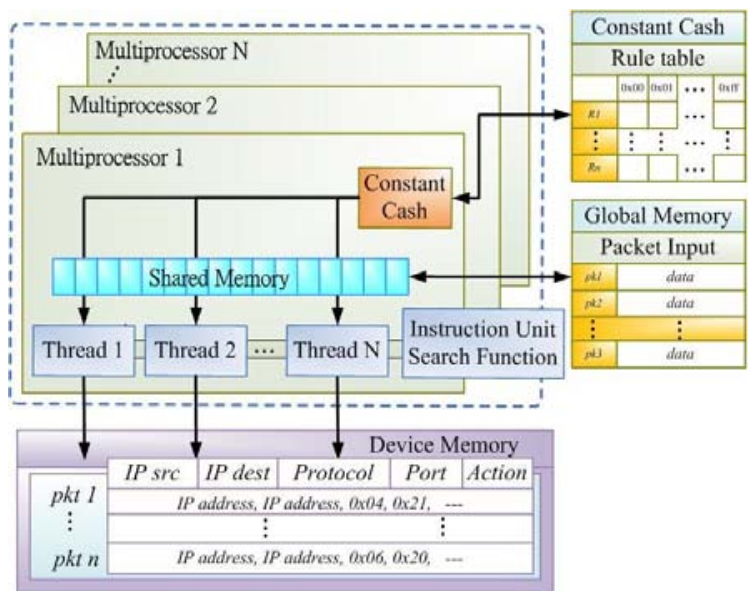

Fig. 4. The memory storage for filter sets (constant memory) and packet data (global memory) on CUDA device.

\subsection{GPU-Based BPF Filtering Algorithm}

BPF was originally proposed for BSD UNIX, it is independent of the TCP/IP stack, and gives user mode processes an interface to access data link layer. It is an elegant and commonly used solution for packet filtering and achieves better perf ormance than other packet filtering systems. B PF consists of two main components: network tap and packet filter ${ }^{22}$. T he network tap is the first interface to copy packets from network interface card driver and moving them to the listening user processes. Then, the packet filter adopts

Table 2. Packet filtering rules

\begin{tabular}{l|l|l|l|l}
\hline Rule & Source IP & Destination IP & Protocol & Port Number \\
\hline 1 & $140.128 .1 .0 /$ & $*$ & $*$ & $*$ \\
& 255.255 .255 .0 & & & \\
2 & $*$ & $140.128 .2 .99 /$ & HTTP & 8888 \\
3 & $140.127 .0 .0 /$ & $125.65 .0 .0 /$ & $*$ & $*$ \\
4 & 255.255 .0 .0 & 255.255 .0 .0 & & $*$ \\
& $219.88 .0 .0 /$ & $123.6 .22 .0 /$ & FTP & $*$ \\
\hline
\end{tabular}


the filtering rules to determine whether a packet should be delivered to the upper-level component or it should be discarded in the kernel space. BPF adopts a directed acyclic control flow graph (CFG) to repres ent a packet filter. Figure 5 shows a sample BPF filter. Each path in the BPF filters represents a comparison procedure needs to be completed for a particular packet pattern. In the proposed GPU-based BPF filtering method, each thread compares the fields of a packet head with the filtering rules. As figure 6, a thread start to do the comparison of the field corresponding to the first condition of rule. If the condition of the rule is $h$ it, the thread then thread stops the comparison and output the results. Otherwise, the thread passes this condition and go to compare next condition. If no condition of a rule is hit, the thread will pass this rule and go to co mpare next rule. Figure 6 shows the proposed GPU-based BPF filtering algorithm. Since GPU has different memory architectures, filtering rules and packet head data can be s tored in these memory architectures shown in Fig. 4.

\subsection{GPU-Based BitMap-RFC Filtering Algorithm}

Different to BPF algorithm, the RFC algorithm is a decomposition-based algorithm that is ab le to provide very high lookup throughput at the cost of low memory efficiency. RFC consists of t wo phases; direct table lookup and crossproducting. In the first phase, it performs the parallel table lookups on each filter field first. This step can achieve the best throughput performance by utilizing a direct table look up. Figure 7 shows the data structure of this table. The table entry $i$ of chunk $j$ stores the set of filters for which the chunk $j$ covers the value $i$. Each unique set of filters is binary encoded. The eqID is the identifier of the encoded set. The second phase is to build a cross-product table with the number of entries equaling to the multiplication of the number of eqIDs of each chunk. However, the number of entries of a cros s-produce table can be very large and it leads to inefficient use of use. To solve this problem, the crossproducting phase is conducted recursively to build multiple cross-product tables.

In the proposed GPU-based BitMap-RFC method, each thread copies with a packet data. In the initial stage, CPU builds all tables, and these tables then are copied to GPU's memory. In the second stage, each thread performs RFC algorithm and store the result to GPU's global memory. After all th reads complete filtering computation, the results will be copy to host's memory.

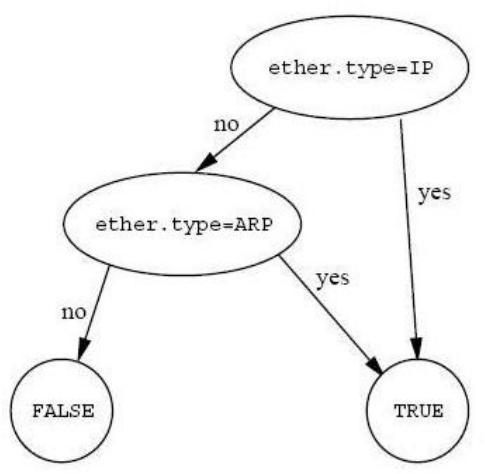

Fig. 5. BPF Filter Model.

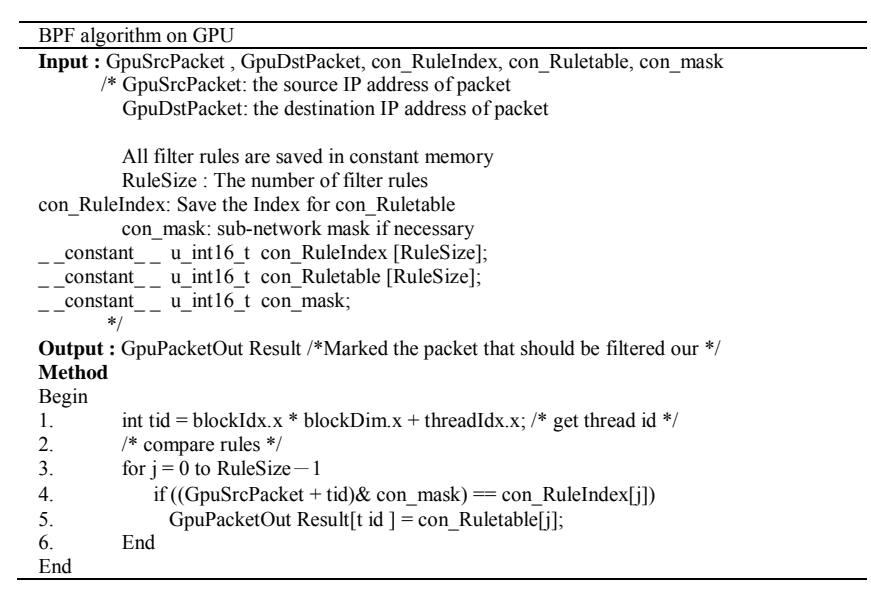

Fig. 6. GPU-based BPF algorithm.

Figure 8 shows the proposed GPU-based BitMap-RFC filtering algorithm. Figure 9 shows the GPU-based packet filter model for BPF and RFC algorithms.

\subsection{GPU Streaming for Implementation of Packet Filter}

In the above section, both of two proposed GPU-based packet filtering methods can adopt direct memory copy and zero-copy models to transfer data to GPU from host and transfer data to host from GPU. In addition, CUDA provides a mechanism, called streaming, to overlap the data transferring and thread computation to enhance the performance. Therefore, the time for transferring data between host and GPU can be decreas ed dramatically. To implement the two proposed methods, all filtering rule tables are co pied to GPU's memory first. The packet data are split in many chunks, and then these chunks are copied t o GPU memory by memory-copy stream sequentially. In the initial phase, the first chunk is copied to GPU's memory completely. Then, these 


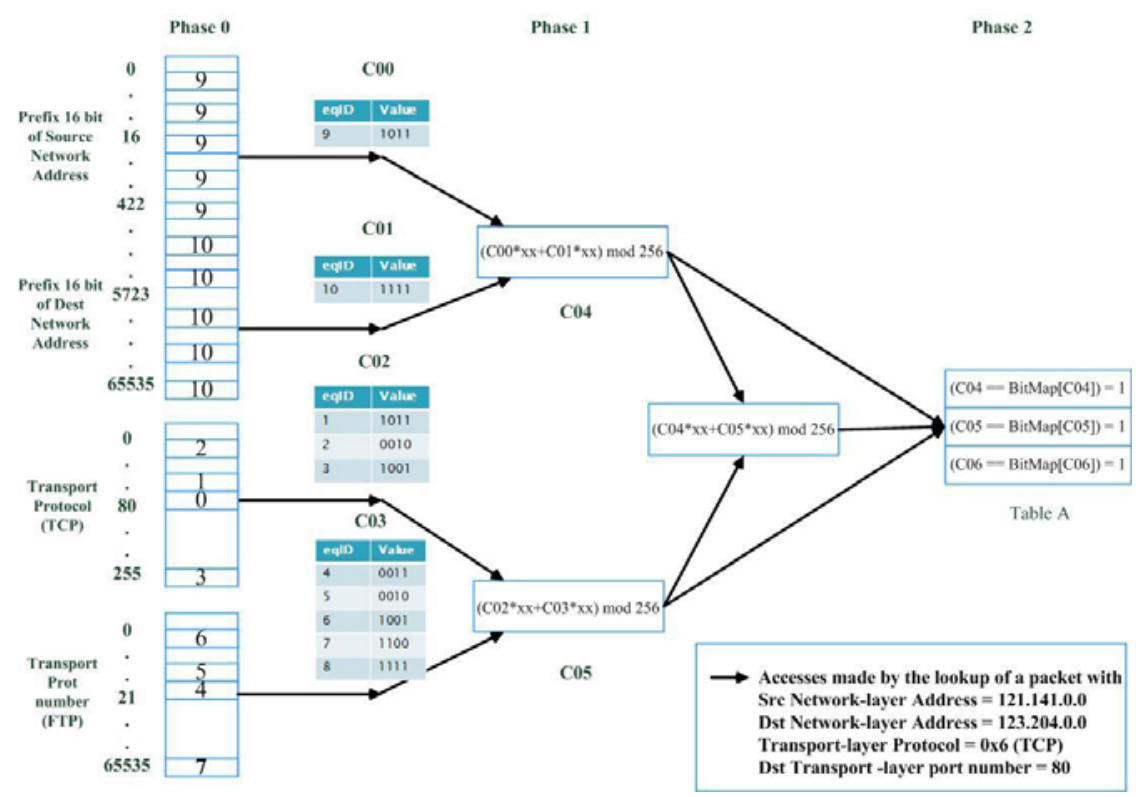

Fig. 7. This is the caption for the figure. If the caption is less than one line then it is centered. Long captions are justified manually.

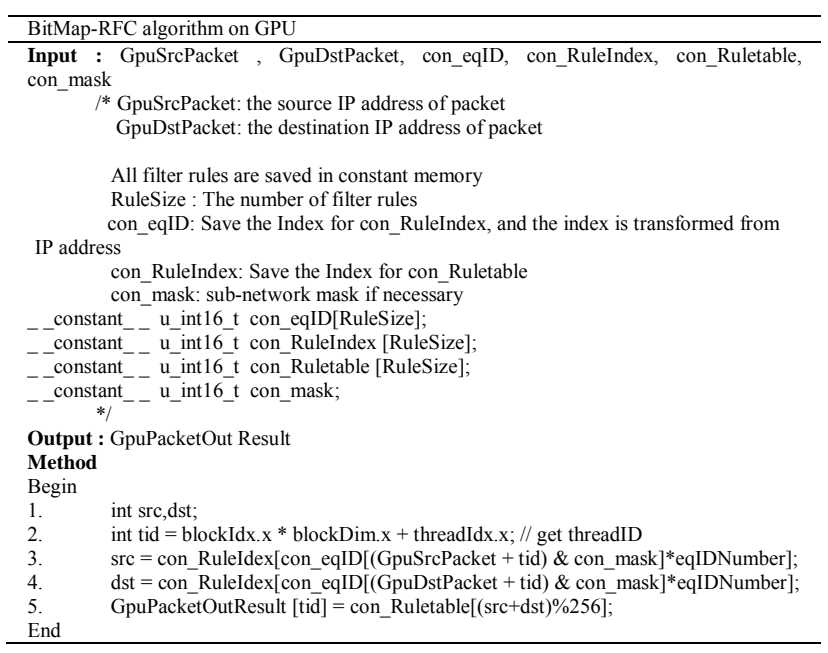

Fig. 8. GPU-based BitMap-RFC algorithm.

packet data is processed and the second chunk is copied to GPU's memory in the second phase. The second phase is repeated until all packet data is $p$ rocessed completely and the filtering results are copied to host's memory.

\section{Experiment}

We implemented two packet classification algorithms, BPF and BitMap-RFC, on single NVIDIA GeForceGTS 560ti graphics card (Fermi architecture) and installed in a PC with an Intel i7-3930k CPU and 16GB DDRIII-

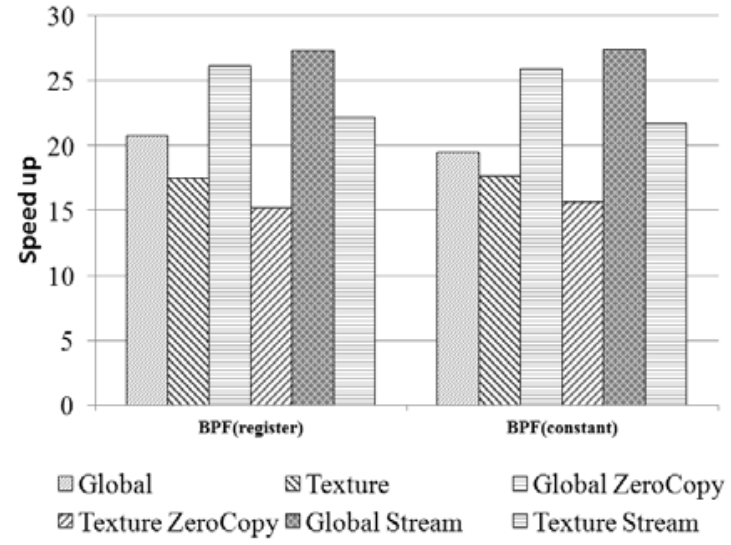

Fig. 9. Performance comparison between CPU-based and GPUbased BPF classification algorithms with a vari ety of memory usage.

1333 RAM running the Linux operating system. We simulated 65 million packets with the random source address, destination address, source port, destination address and protocol for the experiments. The packet filter has three rules and each rule has two fields as shown in Table 2. 1000 classification rules are created by these three rules for the following experiments.

\subsection{Performance Evolution of GPU-Based BPF Packet Filter}

In this experiment, we implemented BPF on CUDA with 10 co mbinations of CUDA memory storages and 


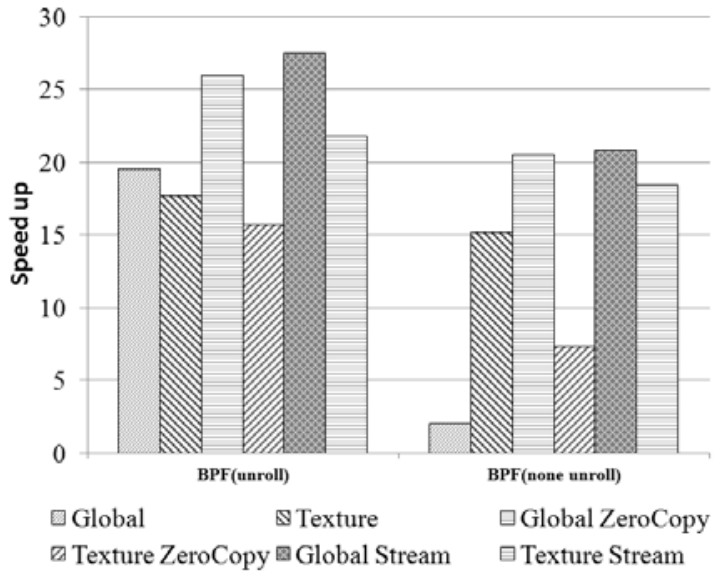

Fig. 10. Performance comparison between GPU-based BPF algorithms with unroll and non-unroll approaches.

data transfer models shown in table 3. Global memory is the biggest memory region available on CUDA devices. Constant memory and register files can access data faster than global and texture memory. However, some limitations on these two structures. First is the storage size. Constant memory is suitable for frequent access but low data update rate. The function of register on CUDA is the same as the registers on CPU. The overusage of register will decrease the performance of GPU. Therefore, we store the packet data in global and texture memory and the filtering rules are sto red in registers and constant memory. Also, we implement the proposed algorithm with three memory copy models, direct copy,

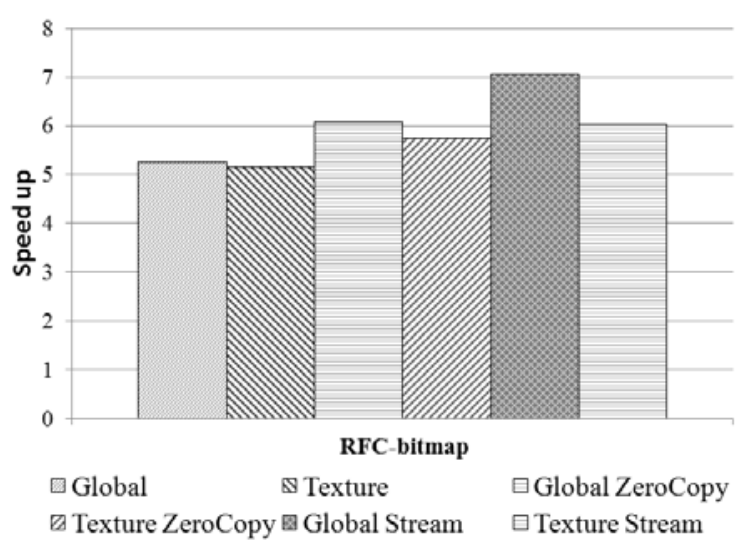

Fig. 11. Performance comparison between CPU-based and GPU-based BitMap-RFC classification algorithms with a variety of memory usage.

zero-copy and streaming.

Figure 9 illustrates the performance comparison between CPU-based and GPU-based BPF classification algorithms. Figure $9 \mathrm{~s}$ hows that the GPU-based BPF algorithm can achieve 20x $\sim 25 \mathrm{x}$ speedup over CPUbased BPF algorithm with storing classification rules in registers for 65 million packets; especially by using streaming model. It is obvious that the speedup of BPF by using global memory with streaming model is slightly superior to that of using global memory with zero-copy.

Actually, we implement GPU-based BPF classification algorithm with an optimization approach,

Table 3. Combination of various memory architectures for GPU-based BPFalgorithm

\begin{tabular}{l|l|l|l}
\hline & Packet Storage & Rule Storage & Data Transfer \\
\hline 1 & Global & Constant & Direct \\
2 & Global & Constant & Zero Copy \\
3 & Texture & Constant & Direct \\
4 & Texture & Constant & Zero Copy \\
5 & Texture & Constant & Stream \\
6 & Global & Register & Direct \\
7 & Global & Register & Zero Copy \\
8 & Texture & Register & Direct \\
9 & Texture & Register & Zero Copy \\
10 & Texture & Register & Stream \\
\hline
\end{tabular}


Table 4. Combination of various memory architectures for GPU-based BitMap-RFC algorithm.

\begin{tabular}{l|l|l|l}
\hline & Packet Location & Rule Location & Data Transfer \\
\hline 1 & Global & Constant & NA \\
2 & Global & Constant & Zero Copy \\
3 & Global & Constant & Stream \\
4 & Texture & Constant & NA \\
5 & Texture & Constant & Zero Copy \\
6 & Texture & Constant & Stream \\
\hline
\end{tabular}

unroll. In the implementation of GPU-based BPF algorithm, lots of loop operations are ad opted to check the filter rules. However, it cau ses a critical $p$ roblem that the number of registers is not enough for computing. The performance is affected. Therefore, an optimization approach, unroll, supported by CUDA is adopted to enhance the performance of GPU-based BPF algorithm. Figure 10 shows the performance between GPU-based BPF with unroll and non-unroll approaches. It is obvious that the performance of GPU-based BPF with unroll is highly superior to that with non-unroll.

\subsection{Performance Evolution of GPU-Based BitMap RFC Packet Filter}

In this experiment, we only implemented BitMap-RFC on CUDA with 6 co mbinations of CUDA memory storages and data transfer models shown in table 4. BitMap-RFC algorithm needs to build hash tables before filtering. Since the sizes of these tables are bigger than that of register files, the filter rules cannot be stored in registers. The results show that the GPU-based RFC algorithm can achieve $5 \mathrm{x} \sim 7 \mathrm{x}$ speedup over CPUbased RFC algorithm in Fig. 11. B ecause CPU-based BitMap-RFC is much faster than CPU-based BPF, the performance enhancement is not dramatic to results in BPF.

\subsection{Performance Evolution between GPU-Based Packet Filter Algorithms}

Figures 12 and 13 show the throughput produced by GPU-based BPF and BitMap-RFC algorithms. Obviously, GPU-based BitMap-RFC algorithm can achieve higher throughput than GPU-based BPF algorithm. The reason is that BPF executes many if-else branch instructions. It cau ses the divergent branch

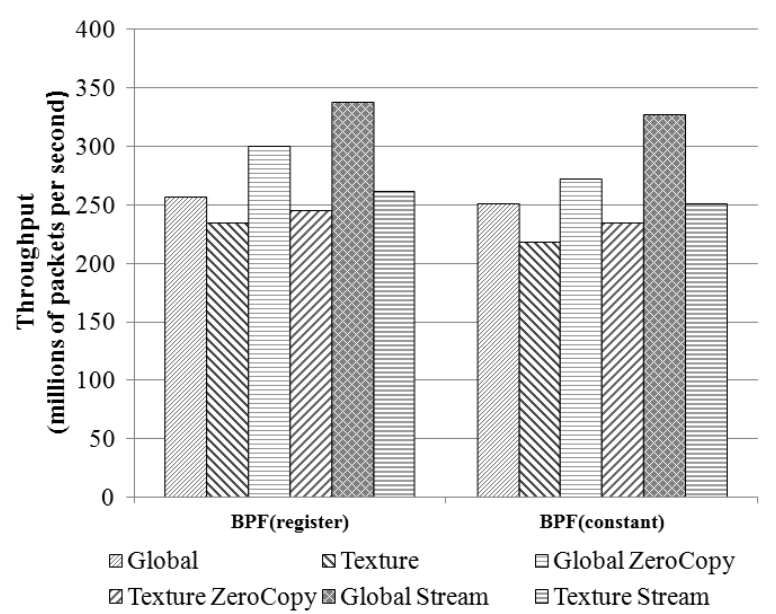

Fig. 12. Throughput comparison between memory usages on GPU-based BPF algorithm.

problem on CUDA device. Therefore, the performance of GPU-based BPF algorithm is decreased by divergent branch problem.

\subsection{Performance Evolution of GPU-Based Packet Filter Algorithms between Different CUDA Devices}

In this experiment, we implemented the proposed algorithms on different CUDA devices, such as GTS 450, GTX480 and GTX560ti. Figure $14 \mathrm{~s}$ hows the throughput comparison between these three CUDA devices. From the result, GTX480 can achieve the highest throughput than other two devices, since GTX480 has more cores than other two devices; GTX480 has 480 cores, GTX450 has 192 cores and GTX560ti has 448 cores. The performance of the proposed algorithms is p roportional to the number of cores of the CUDA device. Therefore, executing the 


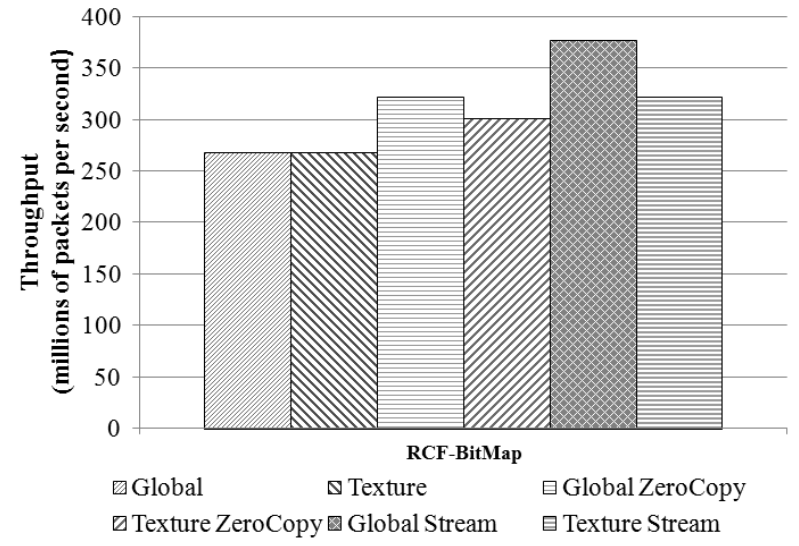

Fig. 13. Throughput comparison between memory usages on GPU-based BitMap-RFC algorithm.

proposed algorithm on high-level CUDA device and the performance can be enhanced.

\section{Conclusion}

In this paper, we propose two fast packet filter methods by leveraging power of GPU device. The proposed methods include two well-know filter algorithms, BPF and BitMap-RFC, to ach ieve rapid and reliable pack et filter system. We implement these algorithms on GPU devices by using CUDA. In addition, we considered the performance of the various memory architectures available to C UDA kernels, and the different data transfer models to enh ance the performance. The experimental results show the performance comparison among several combinations of memory architectures on CUDA device for the proposed methods. Obviously, the proposed methods can significantly enhance the performance over the filter algorithm executing on CPU.

In the future, we will apply GPU-based packet filter system to other network security systems, such as Botnet detection system and network intrusion detection system, to improve the performance. Also, we focus on improving the proposed methods to provide a real-time giga-bit network and fast network telescope packet analysis applications.

\section{Acknowledgements}

This research was partially supported by the National Science Council under the Grants NSC-102-2221-E126-004.

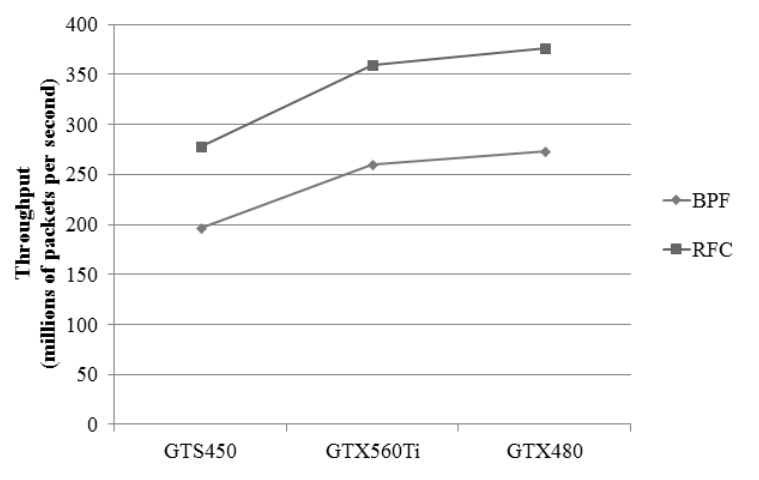

Fig. 14. Throughput comparison between two GPU-based fiilter algorithms on different CUDA devices.

\section{References}

1. D. E. T aylor, Survey and taxonomy of packet classification techniques, ACM Comput. Surv. 37(3) (2005) 238-275.

2. A. Nottingham and B. I rwin, Parallel packet classification using GPU co-processors, SAICSIT Conf. ACM, (2010), pp. 231-24.

3. M. Roesch, Snort - L ightweight intrusion detection for networks, in Proc. the 13th USENIX Conference on System Administration, (1999), pp. 229-238.

4. V. Paxson, Bro: A system for detecting network intruders in real-time, Computer Networks, 31 (1999), pp. 24352463

5. W. Lu, M. Tav.allaee, G. Rammidi and A. A. Ghorbani, Botcop: An online botnet traffic classifier, in Proc. the 7th IEEE Annual Communications Networks and Services Research Conference, (2009), pp. 70-77.

6. F. Alserhani, M. A khlaq, I. U. Awan and A. J. Cullen, Detection of coordinated attacks using alert correlation model, in Proc. IEEE International Conference on Progress in Informatics and Computing, (2010), pp. 542546.

7. M. Szymczyk, Detecting botnets in computer net- works using multi-agent technology, in Proc. the 4th International Conference on Dependability of Computer Systems, (2009), pp. 192-201.

8. L. Braun, G. Munz and G. Carle, Packet sampling for worm and botnet detection in TCP connections, in Proc. IEEE Network Operations and Management Symposium, (2010), pp. 1542-1201.

9. S. Fide and S. Jenks, A Survey of String Matching Approaches in Hardware, Dept. of Electrical Engineering and Computer Science, University of California, Irvine, Tech. Rep. TR SPDS 06-01, (2006).

10. M. Colajanni and M. Marchetti, A Parallel Architecture for Stateful Intrusion Detection in High Traffic Networks, 
in Proc. of Workshop on Monitoring, Attack Detection and Mitigation, (2006).

11. A. Nottingham and B. Irw in, GPU packet classification using OpenCL: a co nsideration of viable classification methods. In Proc. SAICSIT Conf. ACM., (2010), pp. 160169.

12. M. LCharalambous, P. Trancoso and A. Stamatakis, Initial Experiences Porting a Bioinformatics Application to a Graphics Processor, In Proc. the 10th Panhellenic Conference on Informatics, 2005, pp. 415-425.

13. J.D. Owens, D. Luebke, N. Govindaraju, M. Harris, J. Krüger, A.E. Lefohn and T. Purcell, A Survey of General-Purpose Computation on Graphics Hardware, Computer Graphics Forum, 26 (2007), pp. 80-113.

14. C. L. Hung and G. J. Hua, Local Alignment Tool Based on Hadoop Framework and GPU Architecture, Biomed Research International, 2014 (2014), Article ID 541490.

15. S. T. Lee, C. Y. Lin, and C. L. Hung, GPU-Based Cloud Service for Smith-Waterman Algorithm Using Frequency Distance Filtration Scheme, Biomed Research International, 2013 (2013), Article ID 72173.

16. C. Y. Lin, C. L. Hung and Y. C. Hu, A Re-sequencing Tool for High- throughput Long Reads Based on UNImarker with non-Overlapping iNterval indexing strategy, Information - An International Interdisciplinary Journal, 16(1(B)) (2013), pp. 827-832.

17. C. Y. Lin, S. T. Lee and C. L. Hung, Frequency-based resequencing tool for short reads on graphics processing units, International Journal of Computational Science and Engineering, 9(1/2) (2014), pp. 3-10.

18. Nvidia cuda $\mathrm{c}$ best practices guide, version 4. Online, (2011).

19. A. Begel, S. McCanne, and S. L. Graham, BPF+: Exploiting global data-flow optimization in a generalized packet filter architecture, SIGCOMM Comput. Commun. Rev., 29(4) (1999), pp. 123-134.

20. S. Mccanne and V. Jacobson, The bsd packet filter: A new architecture for user-level packet capture, in Proc. the USENIX Winter, (1993), pp. 259-269.

21. E. Spitznagel, D. Taylor, and J. Turner, Packet classification using extended tcams, in Proc. the 11th IEEE International Conference on Network Protocols, (2003), pp. 120-131.

22. S. Singh, F. Baboescu, G. Varghese, and J. Wang, Packet classification using multidimensional cutting, in Proc. the 2003 conference on Applications, technologies, architectures, and protocols for computer communications, (2003), pp. 213-224.

23. V. Srinivasan, G. Varghese, S. Suri, and M. Waldvogel, Fast and scalable layer four switching, SIGCOMM Comput. Commun. Rev., 28(4) (1998), pp. 191-202.

24. D. R. Engler and M. F. Kaashoek, Dpf: Fast, flexible message demultiplexing using dynamic code generation, in Proc. on Applications, technologies, architectures, and protocols for computer communications, (1996), pp. 5359.
25. F. Baboescu and G. Varghese. Scalable packet classification, SIGCOMM Comput. Commun. Rev., 31(4) (2001), pp. 199-210.

26. T. V. L akshman and D. Stiliadis. High-speed policybased packet forwarding using efficient multidimensional range matching, SIGCOMM Comput. Commun. Rev., 28(4) (1998), pp. 203-214.

27. M. Yuhara, B. N. Bershad, C. Maeda, J. Eliot and B. Moss. Efficient packet demultiplexing for multiple endpoints and large messages, In Proceedings of the 1994 Winter USENIX Conference, (1994), pp. 153-165.

28. H. Bos, W. D. Bru ijn, M. Cristea, T. Nguyen and G. Portokalidis, FFPF: Fairly fast packet filters, In Proc. the 6th conference on Symposium on Opearting Systems Design \& Implementation, (2004), pp. 24.

29. S. Ioannidis and K. G. Anagnostakis, XPF: Packet filtering for low-cost network monitoring, In Proc. the IEEE Workshop on High-Performance Switching and Routing, (2002), pp. 121-126.

30. Z. Wu, M. Xie, and H. Wang, Swift: a f ast dynamic packet filter, In Proc. the 5th USENIX Symposium on Networked Systems Design and Implementation, (2008), pp. 279-292.

31. P. Gupta and N. McKeown, Packet Classification on Multiple Fields, SIGCOMM Comput. Commun. Rev., 29 (1999), pp. 147-160.

32. T. Sherwood, G. Varghese and B. Calder, A Pipelined Memory Architecture for High Throughput Network Processors, in Proc. the 30th annual international symposium on Computer architecture, (2003), pp. 288299.

33. M. Degermark, A. Brodnik, S. Carlsson, and S. Pink, Small Forwarding Tables for Fast Routing Lookups, in Proc. ACM SIGCOMM, (1997), pp. 3-14.

34. W. Eatherton, G Varghese, and Z Dittia, Tree Bitmap: Hardware/Software IP Lookups with Incremental Updates, SIGCOMM on Computer Communication Review, 34(2) (2004), pp. 97-122.

35. Xianghui Hu, Xinan Tang, and Bei Hua, A Highperformance IPv6 Forwarding Algorithm for a Multi-core and Multithreaded Network Processor, in Proc. the eleventh ACM SIGPLAN symposium on Principles and practice of parallel programming, (2006), pp. 168-177.

36. E. Spitznagel, Compressed Data Structures for Recursive Flow Classification, Technical Report, WUCSE-2003-65, (2003).

37. D. Liu, B. Hua, X. Hu, and X. Tang, High-performance packet classification algorithm for many-core and multithreaded network processor, in Proc. international conference on Compilers, architecture and synthesis for embedded systems, (2006), pp. 334-344.

38. S. Han, K. Jang, K. Park and S. Moon, PacketShader: a GPU-accelerated Software Router., in Proc. ACM SIGCOMM, (2010), pp. 195-206.

39. C. L. Hung, C. Y. Lin, and H. H. Wang, An Efficient Parallel-Network Packet Pattern-Matching Approach 
Using GPUs, Journal of Systems Architecture, 60(5), (2014), pp. 431-439.

40. N.K. Govindaraju, S. Larsen, J. Gray and D. Manocha, A memory model for scientific algorithms on graphics processors, In Proc. the ACM/IEEE conference on Supercomputing, 2006:89 